\title{
The fractional maximal operator and fractional integrals on variable $L^{p}$ spaces
}

\section{Claudia Capone, David Cruz-Uribe, SFO and Alberto Fiorenza}

\begin{abstract}
We prove that if the exponent function $p(\cdot)$ satisfies log-Hölder continuity conditions locally and at infinity, then the fractional maximal operator $M_{\alpha}, 0<\alpha<n$, maps $L^{p(\cdot)}$ to $L^{q(\cdot)}$, where $\frac{1}{p(x)}-\frac{1}{q(x)}=\frac{\alpha}{n}$. We also prove a weak-type inequality corresponding to the weak $(1, n /(n-\alpha))$ inequality for $M_{\alpha}$. We build upon earlier work on the Hardy-Littlewood maximal operator by Cruz-Uribe, Fiorenza and Neugebauer [3]. As a consequence of these results for $M_{\alpha}$, we show that the fractional integral operator $I_{\alpha}$ satisfies the same norm inequalities. These in turn yield a generalization of the Sobolev embedding theorem to variable $L^{p}$ spaces.
\end{abstract}

\section{Introduction}

Given an open set $\Omega \subset \mathbb{R}^{n}$, and a measurable function $p(\cdot): \Omega \rightarrow[1, \infty)$, let $L^{p(\cdot)}(\Omega)$ denote the Banach function space of measurable functions $f$ on $\Omega$ such that for some $\lambda>0$,

$$
\int_{\Omega}|f(x) / \lambda|^{p(x)} d x<\infty
$$

with norm

$$
\|f\|_{p(\cdot), \Omega}=\inf \left\{\lambda>0: \int_{\Omega}\left(\frac{|f(x)|}{\lambda}\right)^{p(x)} d x \leq 1\right\} .
$$

These spaces are referred to as the variable $L^{p}$ spaces. They are a special case of the Musielak-Orlicz spaces (cf. Musielak [23]), and generalize the classical Lebesgue spaces: when $p(x)=p_{0}$ is constant, $L^{p(\cdot)}(\Omega)=L^{p_{0}}(\Omega)$.

2000 Mathematics Subject Classification: 42B25, $42 \mathrm{~B} 35$.

Keywords: Fractional maximal operator, fractional integral operator, Sobolev embedding theorem, variable Lebesgue space. 
The variable $L^{p}$ spaces are of interest for their applications to modeling problems in physics, and to the study of variational integrals and partial differential equations with non-standard growth conditions. In the past decade they have been considered by a number of authors; see for example $[1,9,11,12,13,17,18,20,22,26,28,31]$ and the references they contain.

We are interested in extending the machinery of classical harmonic analysis to the variable $L^{p}$ spaces. The first step was to determine sufficient conditions on $p(\cdot)$ for the Hardy-Littlewood maximal operator to be bounded on $L^{p(\cdot)}(\Omega)$. Recall that given a locally integrable function $f$, we define the maximal function by

$$
M f(x)=\sup _{B \ni x} \frac{1}{|B|} \int_{B \cap \Omega}|f(y)| d y
$$

where the supremum is taken over all balls $B$ containing $x$. In [3], CruzUribe, Fiorenza and Neugebauer proved the following result. For brevity, hereafter let

$$
p_{-}=\underset{x \in \Omega}{\operatorname{essinf}} p(x), \quad p_{+}=\operatorname{ess} \sup p(x) .
$$

Theorem 1.1. Given an open set $\Omega \subset \mathbb{R}^{n}$, let $p(\cdot): \Omega \rightarrow[1, \infty)$ be such that $1<p_{-} \leq p_{+}<\infty$. Suppose further that $p(\cdot)$ satisfies

$$
|p(x)-p(y)| \leq \frac{C}{-\log |x-y|}, \quad x, y \in \Omega, \quad|x-y|<1 / 2,
$$

and

$$
|p(x)-p(y)| \leq \frac{C}{\log (e+|x|)}, \quad x, y \in \Omega, \quad|y| \geq|x| .
$$

Then the Hardy-Littlewood maximal operator is bounded on $L^{p(\cdot)}(\Omega)$.

Theorem 1.1 was first proved by Diening [5] in the case when $\Omega$ is bounded; in this case only the first continuity condition is needed. He later extended it to unbounded $\Omega$ with the stronger hypothesis that $p(\cdot)$ is constant outside of a large ball. The full result was proved independently by Nekvinda [24] with the second condition replaced by a somewhat more general condition. (See Appendix I below.) Both of these continuity conditions are, in some sense, close to necessary: see the examples in [3] and [25]. Recently, however, Diening [7] discovered a complicated necessary and sufficient condition on the exponent function $p(\cdot)$ for the maximal operator to be bounded on $L^{p(\cdot)}\left(\mathbb{R}^{n}\right)$, which shows that (1.2) and (1.3) are not necessary. In fact, Lerner [21] has constructed an example to show that $p(\cdot)$ does not need to be continuous. 
Remark 1.2. As this paper was being prepared, we discovered a small but significant error in the proof of Theorem 1.1 in [3]. A correction appeared in [4]. In Section 4 we give a simpler proof and discuss the connections with Theorem 1.3 below.

\subsection{The Fractional Maximal Operator}

In this paper we generalize Theorem 1.1 to the fractional maximal operator. Given $\alpha, 0<\alpha<n$, define $M_{\alpha} f$ by

$$
M_{\alpha} f(x)=\sup _{B \ni x} \frac{1}{|B|^{1-\alpha / n}} \int_{B \cap \Omega}|f(y)| d y,
$$

where the supremum is again taken over all balls $B$ which contain $x$. In the limiting case $\alpha=0$, the fractional maximal operator reduces to the HardyLittlewood maximal operator. For $0<\alpha<n, M_{\alpha}$ does not map $L^{p}$ into itself, but instead satisfies the following norm inequality: if $1<p<n / \alpha$ and $q$ is such that $1 / p-1 / q=\alpha / n$, then $M_{\alpha}: L^{p}(\Omega) \rightarrow L^{q}(\Omega)$. If we assume that $p(\cdot)$ satisfies the above continuity conditions, then this result extends to variable $L^{p}$ spaces.

Theorem 1.3. Given an open set $\Omega \subset \mathbb{R}^{n}$ and $\alpha, 0<\alpha<n$, let $p(\cdot): \Omega \rightarrow$ $[1, \infty)$ be such that $1<p_{-} \leq p_{+}<n / \alpha$. Suppose further that $p(\cdot)$ satisfies

$$
|p(x)-p(y)| \leq \frac{C}{-\log |x-y|}, \quad x, y \in \Omega, \quad|x-y|<1 / 2,
$$

and

$$
|p(x)-p(y)| \leq \frac{C}{\log (e+|x|)}, \quad x, y \in \Omega, \quad|y| \geq|x|
$$

Define $q(\cdot): \Omega \rightarrow[1, \infty)$ by

$$
\frac{1}{p(x)}-\frac{1}{q(x)}=\frac{\alpha}{n}, \quad x \in \Omega
$$

Then the fractional maximal operator is bounded from $L^{p(\cdot)}(\Omega)$ to $L^{q(\cdot)}(\Omega)$.

In the setting of classical Lebesgue spaces, Theorem 1.3 follows immediately from the boundedness of the Hardy-Littlewood maximal operator. In fact, using Hölder's inequality it is straightforward to show that

$$
M_{\alpha} f(x) \leq\|f\|_{p, \Omega}^{1-p / q} M f(x)^{p / q}, \quad x \in \Omega .
$$


The desired inequality then follows immediately. When $\Omega$ is bounded this argument can be adapted to the case of variable $L^{p}$ spaces, but inequality (1.5) is false in general. (See Proposition 3.3 and Example 3.4 below.) However, for unbounded sets a weaker version of this inequality still holds (Propositions 3.1 and 3.2 below), and this is sufficient to prove the desired result.

Remark 1.4. Recently, Kokilashvili and Samko [19] proved norm inequalities for a variable fractional maximal operator, with the constant $\alpha$ replaced by a function $\alpha(\cdot)$.

\subsection{Weak-type Inequalities}

We have also generalized the weak-type inequality for the fractional maximal operator. In the classical case, $M_{\alpha}$ is not bounded on $L^{1}$, but the following weak $(1, n /(n-\alpha))$ inequality holds:

$$
\left|\left\{x \in \mathbb{R}^{n}: M_{\alpha} f(x)>t\right\}\right| \leq C\left(\frac{1}{t} \int_{\mathbb{R}^{n}} f(x) d x\right)^{n /(n-\alpha)} .
$$

When $\alpha=0$, Cruz-Uribe, Fiorenza and Neugebauer [4] proved an analog of this inequality. To state it, recall that a function $w \in R H_{\infty}$ if there exists $C>0$ such that for all cubes $Q$,

$$
\frac{C}{|Q|} \int_{Q} w(x) d x \geq \underset{x \in Q}{\operatorname{esssup}} w(x) .
$$

Theorem 1.5. If $p(\cdot): \mathbb{R}^{n} \rightarrow[1, \infty]$ is such that $1 / p(\cdot) \in R H_{\infty}$, then there exists $C>0$ such that for all $t>0$,

$$
\left|\left\{x \in \mathbb{R}^{n}: M f(x)>t\right\}\right| \leq \int_{\mathbb{R}^{n}}\left(\frac{|f(x)|}{t}\right)^{p(x)} d x .
$$

This modular inequality is remarkable since it requires minimal assumptions on $p(\cdot)$ : the $R H_{\infty}$ condition is satisfied if $p_{+}<\infty$, and it holds even for unbounded $p(\cdot)$. However, there does not seem to be a generalization of Theorem 1.5 analogous to (1.6) since in the latter the exponent appears outside the integral.

To avoid this problem we follow a suggestion made by Diening, Hästö and Nekvinda [8] and rewrite (1.6) as a norm inequality. Let $p=1$; then $q=\frac{n}{n-\alpha}$ and (1.6) becomes

$$
\sup _{t>0} t\left\|\chi_{\left\{M_{\alpha} f(x)>t\right\}}\right\|_{q} \leq C\|f\|_{p} .
$$

This inequality has a natural extension to variable $L^{p}$ spaces. 
Theorem 1.6. Given an open set $\Omega \subset \mathbb{R}^{n}$ and $\alpha, 0 \leq \alpha<n$, let $p(\cdot)$ : $\Omega \rightarrow[1, \infty)$ be such that $1=p_{-} \leq p_{+}<n / \alpha$. Suppose further that $p(\cdot)$ satisfies (1.2) and (1.3) and $q(\cdot)$ is defined by (1.4). Then

$$
\sup _{t>0} t\left\|\chi_{\left\{M_{\alpha} f(x)>t\right\}}\right\|_{q(\cdot), \Omega} \leq C\|f\|_{p(\cdot), \Omega} .
$$

Remark 1.7. The key difference in the hypotheses of Theorems 1.3 and 1.6 is that in the latter we need $p_{-}=1$; when $p(\cdot)=1$ then $(1.7)$ reduces to $(1.6)$.

\subsection{Fractional Integrals}

The fractional maximal operator is of interest because it is the natural maximal operator associated with the fractional integral operators (also known as the Riesz potentials). For $0<\alpha<n$, define the fractional integral of order $\alpha$ by

$$
I_{\alpha} f(x)=\int_{\Omega} \frac{f(y)}{|x-y|^{n-\alpha}} d y, \quad x \in \Omega .
$$

As a consequence of Theorems 1.3 and 1.6 and an inequality due to Welland [30] relating $M_{\alpha}$ and $I_{\alpha}$, we prove the following results.

Theorem 1.8. If $\Omega, \alpha, p(\cdot)$, and $q(\cdot)$ are as in Theorem 1.3, then the fractional integral operator $I_{\alpha}$ is bounded from $L^{p(\cdot)}(\Omega)$ to $L^{q(\cdot)}(\Omega)$.

Theorem 1.9. If $\Omega, \alpha, p(\cdot)$, and $q(\cdot)$ are as in Theorem 1.6, then the fractional integral operator $I_{\alpha}$ satisfies the weak-type inequality

$$
\sup _{t>0} t\left\|\chi_{\left\{\left|I_{\alpha} f(x)\right|>t\right\}}\right\|_{q(\cdot), \Omega} \leq C\|f\|_{p(\cdot), \Omega} .
$$

Theorem 1.9 is completely new. The boundedness of $I_{\alpha}$ has been considered by several other authors. Samko [27] proved Theorem 1.8 assuming that $\Omega$ is bounded, $p(\cdot)$ satisfies (1.2), and the maximal operator is bounded. (Note that given Theorem 1.1, his second hypothesis implies his third.)

Theorem 1.8 can also be proved as a consequence of Theorem 1.3 and a result by Diening [6], which is a generalization of an inequality like (1.5) (due to Hedberg [16]) relating $I_{\alpha}$ and the maximal operator. Our approach is somewhat simpler. In the statement of his result Diening assumed that $p(\cdot)$ is constant outside of a large ball, but this stronger hypothesis is only needed to use his version of Theorem 1.1.

Kokilashvili and Samko [19] proved Theorem 1.8 on $\mathbb{R}^{n}$ with $L^{q(\cdot)}$ replaced by a certain weighted variable $L^{p}$ space. (They actually consider a more general operator $I_{\alpha(\cdot)}$ where the constant $\alpha$ in $(1.8)$ is replaced by a function $\left.\alpha(\cdot)\right)$. 


\subsection{The Sobolev Embedding Theorem}

As an immediate application of Theorem 1.8, we extend the Sobolev embedding theorem to variable $L^{p}$ spaces. Given a positive integer $k$, define the variable Sobolev space $W^{k, p(\cdot)}(\Omega)$ to be the Banach space of functions $f$ such that for every multi-index $\alpha$ with $|\alpha| \leq k$, the derivatives $D^{\alpha} f$ (in the sense of distributions) are in $L^{p(\cdot)}(\Omega)$. (See Kováčik and Rákosník [20] for more details.) The classical Sobolev embedding theorem (see, for example, Ziemer [32]) is that if $1<p<n / k$ and $\Omega$ is an extension domain, then $W^{k, p}(\Omega) \subset L^{q}(\Omega)$, where $1 / q=1 / p-k / n$. We generalize this result in the special case when $\Omega=\mathbb{R}^{n}$.

Theorem 1.10. Let $p(\cdot): \mathbb{R}^{n} \rightarrow[1, \infty)$ be such that $1<p_{-} \leq p_{+}<n$ and $(1.2)$ and (1.3) hold. If $k$ is an integer such that $p_{+}<n / k$, and if we define $q(\cdot)$ by

$$
\frac{1}{q(x)}=\frac{1}{p(x)}-\frac{k}{n}, \quad x \in \mathbb{R}^{n}
$$

then $W^{k, p(\cdot)}\left(\mathbb{R}^{n}\right) \subset L^{q(\cdot)}\left(\mathbb{R}^{n}\right)$.

Theorem 1.10 follows immediately from Theorem 1.8, and the proof is essentially identical to the proof in the classical setting - see, for instance, Ziemer [32, p. 88]. This proof assumes that $C_{c}^{\infty}\left(\mathbb{R}^{n}\right)$ is dense in $W^{k, p(\cdot)}\left(\mathbb{R}^{n}\right)$, but this is the case since $p(\cdot)$ satisfies (1.2) and (1.3). (See Samko [28], Diening [6], or [2].)

Diening [6] gave essentially the same proof of Theorem 1.10; again he assumed that $p(\cdot)$ is bounded outside of a large ball in order to use his version of Theorem 1.1.

There have been many attempts to extend the Sobolev embedding theorem to variable $L^{p}$ spaces. Kováčik and Rákosník [20] proved a weak version, assuming that the domain $\Omega$ is bounded and $p(\cdot)$ is continuous on $\bar{\Omega}$, but with $L^{q(\cdot)}$ replaced by $L^{q(\cdot)-\epsilon}$ for any $\epsilon>0$. Edmunds and Rákosník [9] proved a version assuming that $\Omega$ is bounded and has Lipschitz boundary, and $p(\cdot)$ is a Lipschitz continuous function on $\bar{\Omega}$. More recently [10] they improved their result by showing they could assume that $p(\cdot) \in W^{1, s}(\Omega)$, $n<s<\infty$, which in turn implies that $p(\cdot)$ is Hölder continuous (see Gilbarg and Trudinger [14, section 7.7]). Finally, Diening [[6], Corollary 5.3] proved the more general embedding theorem when $\Omega$ is bounded and has Lipschitz boundary, assuming only that $p(\cdot)$ satisfies $(1.2)$. 


\subsection{Organization}

The rest of this paper is organized as follows. In Section 2 we gather some preliminary lemmas about variable $L^{p}$ spaces. The heart of the paper is Section 3 where we prove Theorem 1.3. In Sections 4 and 5 we prove Theorem 1.6. In Section 4 we prove an inequality for averages over balls which lies at the heart of the proof. As a corollary we are able to give a new proof of Theorem 1.1 which in turn leads to a conjecture related to the proof of Theorem 1.3. In Section 5 we use this inequality to prove Theorem 1.6. In Section 6 we prove Theorems 1.8 and 1.9. Finally, in Appendix A we consider the relationship between condition (1.3) and the more general condition (given for the case $\Omega=\mathbb{R}^{n}$ ) introduced by Nekvinda [24].

Throughout the paper, notation will be standard or defined as needed. We will assume that the reader is familiar with the basic properties of variable $L^{p}$ spaces, and refer to Kováčik and Rákosník [20] for more information. In order to emphasize that we are dealing with variable exponents, we will always write $p(\cdot)$ instead of $p$ to denote an exponent function. Given an open set $\Omega$ and function $p(\cdot), 1 \leq p(x) \leq \infty, x \in \Omega$, define the conjugate function $p^{\prime}(\cdot)$ to satisfy $1 / p(x)+1 / p^{\prime}(x)=1$, where we take $1 / \infty=0$. Given a set $E$, let $|E|$ denote its Lebesgue measure, and let $p_{-}(E)=\operatorname{ess} \inf \{p(y): y \in E\}$ and $p_{+}(E)=\operatorname{ess} \sup \{p(y): y \in E\}$. For brevity, let $p_{-}=p_{-}(\Omega)$ and $p_{+}=p_{+}(\Omega)$. Given a function $f$, let

$$
|f|_{p(\cdot), \Omega}=\int_{\Omega}|f(y)|^{p(y)} d y .
$$

Finally, $C$ and $c$ will denote positive constants which will depend only on the dimension $n$, the underlying set $\Omega$ and the exponent function $p(\cdot)$, but whose value may change at each appearance.

\section{Preliminary Results}

In this section we gather together a number of lemmas which we need in the next section. First, we note that while the definition of the norm in variable $L^{p}$ spaces given at the beginning of the Introduction is suitable for the statement of our results, we need a somewhat more general definition in our proofs.

Definition 2.1. Given an open set $\Omega \subset \mathbb{R}^{n}$, and a measurable function $p(\cdot): \Omega \rightarrow[1, \infty]$, we say $f \in L^{p(\cdot)}(\Omega)$ if for some $\lambda>0$,

$$
\rho(p(\cdot), \Omega, f / \lambda)=\int_{\Omega \backslash \Omega_{\infty}}|f(x) / \lambda|^{p(x)} d x+\|f / \lambda\|_{\infty, \Omega_{\infty}}<\infty,
$$

where $\Omega_{\infty}=\{x \in \Omega: p(x)=\infty\}$. The norm on $L^{p(\cdot)}(\Omega)$ is given by

$$
\|f\|_{p(\cdot), \Omega}=\inf \{\lambda>0: \rho(p(\cdot), \Omega, f / \lambda) \leq 1\} .
$$


Next, we give three basic results about variable $L^{p}$ spaces. Note in particular that the second one depends on Definition 2.1. Their proofs can be found in Kováčik and Rákosník [20].

Lemma 2.2. Given $p(\cdot): \Omega \rightarrow[1, \infty)$ such that $p_{+}<\infty$, then $\|f\|_{p(\cdot), \Omega}<C_{1}$ if and only if $|f|_{p(\cdot), \Omega}<C_{2}$. In particular, if either constant equals 1 we can take the other equal to 1 as well.

Lemma 2.3. Given an open set $\Omega$ and $p(\cdot): \Omega \rightarrow[1, \infty]$, we have that for all functions $f$ and $g$,

$$
\int_{\Omega}|f(x) g(x)| d x \leq C\|f\|_{p(\cdot), \Omega}\|g\|_{p^{\prime}(\cdot), \Omega} .
$$

Lemma 2.4. Given a set $\Omega$ with finite measure, and exponent functions $p(\cdot), q(\cdot): \Omega \rightarrow[1, \infty]$ such that $p(x) \leq q(x)$,

$$
\|f\|_{p(\cdot), \Omega} \leq C(1+|\Omega|)\|f\|_{q(\cdot), \Omega} .
$$

The next lemma is due to Diening [5]; a proof is also found in [3]. It is through this lemma that we see the connection between (1.2) and maximal operators.

Lemma 2.5. Given an open set $\Omega$ and a function $p(\cdot): \Omega \rightarrow[1, \infty)$ which satisfies (1.2), then for any ball $B$ such that $|B \cap \Omega|>0$,

$$
|B|^{p_{-}(B \cap \Omega)-p_{+}(B \cap \Omega)} \leq C .
$$

The next lemma is a variant of a lemma due to Diening [6]. For the convenience of the reader we include its short proof.

Lemma 2.6. Given an open set $\Omega$ and a function $p(\cdot): \Omega \rightarrow[1, \infty), p_{+}<\infty$, which satisfies (1.2), then for any ball $B$ such that $|B \cap \Omega|>0$ and $|B| \leq 1$,

$$
\left\|\chi_{B \cap \Omega}\right\|_{p(\cdot), \Omega} \leq C|B|^{1 / p(x)} .
$$

Proof. Since $p_{+}<\infty$, by the definition of the norm on $L^{p(\cdot)}(\Omega)$, and since $|B| \leq 1$,

$$
\begin{aligned}
\left\|\chi_{B \cap \Omega}\right\|_{p(\cdot), \Omega} & =\inf \left\{\lambda>0: \int_{B \cap \Omega} \lambda^{-p(x)} d x \leq 1\right\} \\
& =\inf \left\{0<\lambda<1: \int_{B \cap \Omega} \lambda^{-p(x)} d x \leq 1\right\} \\
& \leq \inf \left\{0<\lambda<1: \int_{B} \lambda^{-p_{+}(B \cap \Omega)} d x \leq 1\right\}=|B|^{1 / p_{+}(B \cap \Omega)} .
\end{aligned}
$$

By Lemma 2.5,

$$
\begin{aligned}
|B|^{1 / p_{+}(B \cap \Omega)} & =|B|^{1 / p(x)}|B|^{1 / p_{+}(B \cap \Omega)-1 / p(x)} \\
& \leq|B|^{1 / p(x)}|B|^{p_{-}\left((B \cap \Omega)-p_{+}(B \cap \Omega)\right) / p_{-}^{2}} \leq C|B|^{1 / p(x)} .
\end{aligned}
$$


The next two lemmas allow one exponent function to be substituted for another; it is here that (1.3) plays a central role. The first is a variation of a result in [3] that was given in [4]; for completeness we include its short proof. Here and below, for $t>0$ let $R_{t}(x)=(e+|x|)^{-t n}$.

Lemma 2.7. Given a set $G$ and two non-negative functions $r(\cdot)$ and $s(\cdot)$, suppose that for each $y \in G$,

$$
0 \leq s(y)-r(y) \leq \frac{C}{\log (e+|z(y)|)},
$$

where $z: G \rightarrow \mathbb{R}^{n}$. Then there exists a constant $C_{t}$ such that for every function $f$,

$$
\int_{G}|f(y)|^{r(y)} d y \leq C_{t} \int_{G}|f(y)|^{s(y)} d y+\int_{G} R_{t}(z(y))^{r_{-}(G)} d y .
$$

Proof. Let $G^{R_{t}}=\left\{y \in G:|f(y)| \geq R_{t}(z(y))\right\}$. Then

$$
\int_{G}|f(y)|^{r(y)} d y=\int_{G^{R_{t}}}|f(y)|^{r(y)} d y+\int_{G \backslash G^{R_{t}}}|f(y)|^{r(y)} d y,
$$

and we estimate each integral separately. First, since $R_{t}(z(y)) \leq 1$,

$$
\int_{G \backslash G^{R_{t}}}|f(y)|^{r(y)} d y \leq \int_{G \backslash G^{R_{t}}} R_{t}(z(y))^{r(y)} d y \leq \int_{G} R_{t}(z(y))^{r_{-}(G)} d y .
$$

On the other hand, if $y \in G^{R_{t}}$, then

$$
\begin{aligned}
|f(y)|^{r(y)} & =|f(y)|^{s(y)}|f(y)|^{r(y)-s(y)} \\
& \leq|f(y)|^{s(y)} R_{t}(z(y))^{-C / \log (e+|z(y)|)} \leq C_{t}|f(y)|^{s(y)} .
\end{aligned}
$$

The desired inequality now follows immediately.

The second lemma is a variation of Lemma 2.7; its proof is the same, mutatis mutandis.

Lemma 2.8. Given a set $G$ and two non-negative functions $r(\cdot)$ and $s(\cdot)$, suppose that for each $y \in G$,

$$
|s(y)-r(y)| \leq \frac{C}{\log (e+|y|)}
$$

Then there exists a constant $C_{t}$ such that for every function $f$ such that $|f(y)| \leq 1, y \in G$,

$$
\int_{G}|f(y)|^{r(y)} d y \leq C_{t} \int_{G}|f(y)|^{s(y)} d y+\int_{G} R_{t}(y)^{r_{-}(G)} d y .
$$




\section{Proof of Theorem 1.3}

In this section we prove Theorem 1.3. At the heart of our argument are the following two propositions; they generalize inequality (1.5) and the main lemmas in [3] and [5]. Their proofs are somewhat technical, so we defer them until after the proof of our main result.

Proposition 3.1. Given an open set $\Omega \subset \mathbb{R}^{n}$, and $\alpha, 0<\alpha<n$, let $p(\cdot): \Omega \rightarrow[1, \infty)$ be such that $1 \leq p_{-} \leq p_{+}<n / \alpha$ and such that $(1.2)$ holds. Let $q(\cdot)$ be given by (1.4). Then for all $f \in L^{p(\cdot)}(\Omega)$ such that $\|f\|_{p(\cdot), \Omega} \leq 1$ and such that $|f(x)| \geq 1$ or $f(x)=0, x \in \Omega$,

$$
M_{\alpha} f(x) \leq C M f(x)^{p(x) / q(x)} .
$$

To state the next proposition, we need a definition. Given any exponent function $r(\cdot): \Omega \rightarrow[1, \infty)$, define

$$
I_{r}(x)=\sup _{|y| \geq|x|} r(y)
$$

Proposition 3.2. Given an open set $\Omega \subset \mathbb{R}^{n}$, and $\alpha, 0<\alpha<n$, let $p(\cdot): \Omega \rightarrow[1, \infty)$ be such that $1 \leq p_{-} \leq p_{+}<n / \alpha$ and such that $(1.3)$ holds. Let $q(\cdot)$ be given by (1.4). Then for all $f \in L^{p(\cdot)}(\Omega)$ such that $\|f\|_{p(\cdot), \Omega} \leq 1$ and such that $|f(x)| \leq 1, x \in \Omega$,

$$
M_{\alpha} f(x) \leq C M f(x)^{p(x) / I_{q}(x)} .
$$

Proof of Theorem 1.3. Fix $f \in L^{p(\cdot)}(\Omega)$; without loss of generality we may assume that $f$ is non-negative and that $\|f\|_{p(\cdot), \Omega} \leq 1$. By Lemma 2.2 it will suffice to show that $\left|M_{\alpha} f\right|_{q(\cdot), \Omega} \leq C$. Further, since the hypotheses of Theorem 1.1 are satisfied, $\|M f\|_{p(\cdot), \Omega} \leq C\|f\|_{p(\cdot), \Omega} \leq C$. Therefore, again by Lemma 2.2 we have that $|M f|_{p(\cdot), \Omega} \leq C$.

Define $f_{1}=f \chi_{\{x: f(x) \geq 1\}}$ and $f_{2}=f-f_{1}$. Then $\left\|f_{i}\right\|_{p(\cdot), \Omega} \leq\|f\|_{p(\cdot), \Omega}=1$, $i=1,2$. Therefore, since $q_{+}<\infty$, and by Propositions 3.1 and 3.2,

$$
\begin{aligned}
\int_{\Omega} M_{\alpha} f(x)^{q(x)} d x & \leq 2^{q_{+}} \int_{\Omega} M_{\alpha} f_{1}(x)^{q(x)} d x+2^{q_{+}} \int_{\Omega} M_{\alpha} f_{2}(x)^{q(x)} d x \\
& \leq C \int_{\Omega} M f_{1}(x)^{p(x)} d x+C \int_{\Omega} M f_{2}(x)^{p(x) q(x) / I_{q}(x)} d x .
\end{aligned}
$$

We estimate each integral in turn. The first is immediate:

$$
C \int_{\Omega} M f_{1}(x)^{p(x)} d x \leq C \int_{\Omega} M f(x)^{p(x)} d x \leq C .
$$


To estimate the second, we first need to control the quantity $q(x) / I_{q}(x)$. Fix $\epsilon>0$. Then there exists $y,|y| \geq|x|$ such that $I_{q}(x) \leq(1+\epsilon) q(y)$. Then by $(1.3)$,

$0 \leq I_{q}(x)-q(x) \leq(1+\epsilon) q(y)-q(x) \leq|q(y)-q(x)|+\epsilon q_{+} \leq \frac{C}{\log (e+|x|)}+\epsilon q_{+}$.

Since $\epsilon>0$ is arbitrary and $I_{q}(x) \geq 1$, it follows that

$$
0 \leq 1-q(x) / I_{q}(x) \leq \frac{C}{\log (e+|x|)} .
$$

Let $r(x)=q(x) / I_{q}(x)$. Then $r_{-} \geq q_{-} / q_{+}$, so $R_{2 q_{+} / q_{-}}(\cdot)^{r_{-}}$is integrable. Therefore, by Lemma 2.7 (with $s(x)=1$ ),

$$
\int_{\Omega} M f_{2}(x)^{p(x) q(x) / I_{q}(x)} d x \leq C_{t} \int_{\Omega} M f(x)^{p(x)} d x+\int_{\Omega} R_{2 q_{+} / q_{-}}(x)^{r_{-}} d x \leq C .
$$

This completes the proof.

Proof of Proposition 3.1. Fix $x \in \Omega$, and fix a ball $B$ containing $x$. Then by the definition of $q(\cdot)$,

$$
\begin{aligned}
& \frac{1}{|B|^{1-\alpha / n}} \int_{B \cap \Omega}|f(y)| d y \\
& \quad=|B|^{\alpha / n}\left(\frac{1}{|B|} \int_{B \cap \Omega}|f(y)| d y\right)^{\alpha p(x) / n}\left(\frac{1}{|B|} \int_{B \cap \Omega}|f(y)| d y\right)^{1-\alpha p(x) / n} \\
& \quad \leq|B|^{\alpha / n}\left(\frac{1}{|B|} \int_{B \cap \Omega}|f(y)| d y\right)^{\alpha p(x) / n} M f(x)^{p(x) / q(x)} .
\end{aligned}
$$

To complete the proof we will show that

$$
|B|^{\alpha / n}\left(\frac{1}{|B|} \int_{B \cap \Omega}|f(y)| d y\right)^{\alpha p(x) / n} \leq C\|f\|_{p(\cdot), \Omega}^{1-p(x) / q(x)} \leq C .
$$

There are two cases depending on the size of $B$. Suppose first that $|B| \geq 1$. Let $\Omega_{f}=\operatorname{supp}(f)$. By Chebyschev's inequality and Lemma 2.2,

$$
\left|\Omega_{f}\right| \leq|f|_{p(x), \Omega} \leq\|f\|_{p(x), \Omega} \leq 1 .
$$

Therefore, since $p(x) \geq 1$, by Lemma 2.4 ,

$$
\begin{aligned}
|B|^{\alpha / n}\left(\frac{1}{|B|} \int_{B \cap \Omega}|f(y)| d y\right)^{\alpha p(x) / n} & \leq\left(\int_{B \cap \Omega}|f(y)| d y\right)^{\alpha p(x) / n} \\
& \leq\|f\|_{1, \Omega_{f}}^{\alpha p(x) / n} \\
& \leq\left(C\left(1+\left|\Omega_{f}\right|\right)\|f\|_{p(\cdot), \Omega_{f}}\right)^{\alpha p(x) / n} \\
& \leq C .
\end{aligned}
$$


Now suppose $|B| \leq 1$. If $p_{-}(B \cap \Omega)>1$, then $\left(p^{\prime}\right)_{+}(B \cap \Omega)<\infty$ and $p^{\prime}(\cdot)$ satisfies $(1.2)$, so by Lemmas 2.3 and 2.6 ,

$$
\begin{aligned}
|B|^{\alpha / n}\left(\frac{1}{|B|} \int_{B \cap \Omega}|f(y)| d y\right)^{\alpha p(x) / n} & \leq|B|^{\alpha / n-\alpha p(x) / n}\left\|\chi_{B \cap \Omega}\right\|_{p^{\prime}(\cdot), \Omega}^{\alpha p(x) / n}\|f\|_{p(\cdot), \Omega}^{\alpha p(x) / n} \\
& \leq|B|^{\alpha / n-\alpha p(x) / n}\left\|\chi_{B \cap \Omega}\right\|_{p^{\prime}(\cdot), \Omega}^{\alpha p(x) / n} \\
& \leq C|B|^{\alpha / n-\alpha p(x) / n}|B|^{\frac{\alpha p(x)}{n p^{\prime}(x)}} \\
& \leq C .
\end{aligned}
$$

The argument is the same when $p_{-}(B \cap \Omega)=1$ except that instead of applying Lemma 2.6 we note that by Lemma 2.4 ,

$$
\left\|\chi_{B \cap \Omega}\right\|_{p^{\prime}(\cdot), \Omega}^{\alpha p(x) / n}=\left\|\chi_{B \cap \Omega}\right\|_{p^{\prime}(\cdot), B \cap \Omega}^{\alpha p(x) / n} \leq C(1+|B|)\left\|\chi_{B \cap \Omega}\right\|_{\infty, B \cap \Omega}^{\alpha p(x) / n} \leq C .
$$

Then by Lemma 2.5,

$$
|B|^{\alpha / n-\alpha p(x) / n} \leq|B|^{\left(p_{-}(B \cap \Omega)-p_{+}(B \cap \Omega)\right) \alpha / n} \leq C .
$$

This completes the proof.

The above argument readily adapts to prove that (1.5) holds in variable $L^{p}$ spaces if we assume that $\Omega$ is bounded. Details of the proof are left to the reader.

Proposition 3.3. Given a bounded, open set $\Omega \subset \mathbb{R}^{n}$, and $\alpha, 0<\alpha<n$, let $p(\cdot): \Omega \rightarrow[1, \infty)$ be such that $1<p_{-} \leq p_{+}<n / \alpha$ and such that $(1.2)$ holds. Let $q(\cdot)$ be given by (1.4). Then for all $f \in L^{p(\cdot)}(\Omega)$,

$$
M_{\alpha} f(x) \leq C(\Omega, n, p(\cdot))\|f\|_{p(\cdot), \Omega}^{1-p(x) / q(x)} M f(x)^{p(x) / q(x)}, \quad x \in \Omega .
$$

When $\Omega$ is unbounded (3.3) need not hold, even if $p(\cdot)$ satisfies (1.2) and (1.3).

Example 3.4. There exists an exponent function $p(\cdot):[1, \infty) \rightarrow \mathbb{R}$ such that (1.2) and (1.3) hold but such that (3.3) does not hold uniformly for all $f \in L^{p(\cdot)}(\mathbb{R})$.

Proof. Let $\Omega=\mathbb{R}, \alpha=1 / 4$, and let $p(\cdot)$ be any smooth exponent function such that $p(0)=2$ and $p(x)=3$ if $|x| \geq 1$. Define the sequence of functions $\left\{f_{n}\right\}_{1}^{\infty}$ by $f_{n}(x)=n^{-1} \chi_{[n, 2 n]}$. We will show that inequality (3.3) does not 
hold with a uniform constant for this family. Defining $q(\cdot)$ as above, we have that $q(0)=4$. Straightforward computations show that

$$
\begin{gathered}
\left\|f_{n}\right\|_{p(\cdot), \mathbb{R}}=n^{-2 / 3}, \\
M f_{n}(0)=\frac{1}{2 n} \int_{0}^{2 n}\left|f_{n}(y)\right| d y=(2 n)^{-1}, \\
M_{\alpha} f_{n}(0) \geq \frac{1}{(2 n)^{3 / 4}} \int_{0}^{2 n}\left|f_{n}(y)\right| d y=(2 n)^{-3 / 4} .
\end{gathered}
$$

Therefore, if (3.3) held, then when $x=0$ we would have that for all $n$,

$$
(2 n)^{-3 / 4} \leq C n^{-(2 / 3)(1-2 / 4)}(2 n)^{-2 / 4}=C n^{-5 / 6},
$$

which is clearly impossible.

Proof of Proposition 3.2. Fix $x \in \Omega$ and let $B$ be any ball containing $x$. It will suffice to show that

$$
\frac{1}{|B|^{1-\alpha / n}} \int_{B \cap \Omega}|f(y)| d y \leq C M f(x)^{p(x) / I_{q}(x)},
$$

where $C$ is independent of $B$.

It follows at once from the definition of $q(\cdot)$ that $1 / I_{p}(x)-1 / I_{q}(x)=\alpha / n$. Therefore, by Hölder's inequality,

$$
\begin{aligned}
& \frac{1}{|B|^{1-\alpha / n}} \int_{B \cap \Omega}|f(y)| d y \\
& \quad=|B|^{\alpha / n}\left(\frac{1}{|B|} \int_{B \cap \Omega}|f(y)| d y\right)^{\alpha I_{p}(x) / n}\left(\frac{1}{|B|} \int_{B \cap \Omega}|f(y)| d y\right)^{1-\alpha I_{p}(x) / n} \\
& \quad \leq\left(\int_{B \cap \Omega}|f(y)|^{I_{p}(x)} d y\right)^{\alpha / n} M f(x)^{I_{p}(x) / I_{q}(x)}
\end{aligned}
$$

Since $|f(x)| \leq 1, M f(x) \leq 1$, so $M f(x)^{I_{p}(x) / I_{q}(x)} \leq M f(x)^{p(x) / I_{q}(x)}$. Therefore, to complete the proof we only need to show that the last integral is bounded by a constant. Define the sets

$$
\begin{aligned}
& B_{x}=\{y \in B \cap \Omega,|y| \leq|x|\}, \\
& B^{x}=\{y \in B \cap \Omega,|y|>|x|\} .
\end{aligned}
$$

Then

$$
\int_{B \cap \Omega}|f(y)|^{I_{p}(x)} d y=\int_{B_{x}}|f(y)|^{I_{p}(x)} d y+\int_{B^{x}}|f(y)|^{I_{p}(x)} d y
$$


The second integral is easy to estimate. If $y \in B^{x}$ then $I_{p}(x) \geq p(y)$; thus, since $|f(y)| \leq 1$, by Lemma 2.2 ,

$$
\int_{B^{x}}|f(y)|^{I_{p}(x)} d y \leq \int_{B^{x}}|f(y)|^{p(y)} d y \leq|f|_{p(\cdot), \Omega} \leq 1 .
$$

To estimate the first integral we will apply Lemma 2.8. If $y \in B_{x}$, then by (1.3),

$$
\left|p(y)-I_{p}(x)\right| \leq \frac{C}{\log (e+|y|)}
$$

Therefore, for any $t>1$,

$$
\int_{B_{x}}|f(y)|^{I_{p}(x)} d y \leq C_{t} \int_{B_{x}}|f(y)|^{p(y)} d y+\int_{B_{x}} R_{t}(y)^{\left(I_{p}\right)} d y \leq C .
$$

This completes the proof.

\section{An Inequality for the Maximal Operator}

In this section we prove the following inequality for averages over balls.

Theorem 4.1. Given an open set $\Omega \subset \mathbb{R}^{n}$, let $p(\cdot): \Omega \rightarrow[1, \infty)$ satisfy $(1.2)$ and (1.3). Suppose $f \in L^{p(\cdot)}(\Omega)$ is such that $|f|_{p(\cdot), \Omega} \leq 1$. Then for every ball $B$ and every $x \in B$,

$$
\left(\frac{1}{|B|} \int_{B \cap \Omega}|f(y)| d y\right)^{p(x)} \leq C\left(\frac{1}{|B|} \int_{B \cap \Omega}|f(y)|^{p(y) / p_{-}} d y\right)^{p_{-}}+S(x)
$$

where $S(\cdot) \in L^{1}\left(\mathbb{R}^{n}\right)$ and $C=C(n, p(\cdot))$.

Before proving Theorem 4.1 we first want to make some observations. As an immediate consequence of it, we have the following pointwise inequality for the maximal operator:

$$
M f(x)^{p(x)} \leq C M\left(|f(\cdot)|^{p(\cdot) / p_{-}}\right)(x)^{p_{-}}+S(x) .
$$

Inequality (4.2) is a significantly simplified version of the two key lemmas in [3]; given it, the proof of Theorem 1.1 is straightforward. Fix $f \in L^{p(\cdot)}(\Omega)$; without loss of generality we may assume that $\|f\|_{p(\cdot), \Omega} \leq 1$. By Lemma 2.2 it will suffice to show that $|M f|_{p(\cdot), \Omega} \leq C$. Since $\|f\|_{p(\cdot), \Omega} \leq 1$, again by Lemma 2.2 we have that $|f|_{p(\cdot), \Omega} \leq 1$. If we integrate (4.2) over $\Omega$, we get

$$
\int_{\Omega} M f(x)^{p(x)} d x \leq C \int_{\Omega} M\left(|f(\cdot)|^{p(\cdot) / p_{-}}\right)(x)^{p_{-}} d x+\int_{\Omega} S(x) d x .
$$


Since $S \in L^{1}(\Omega)$, the second integral is bounded by a constant; since $p_{-}>1$, the maximal operator is bounded on $L^{p_{-}}(\Omega)$ (see, for example, Stein $[29$, p. 5]). Thus,

$$
\int_{\Omega} M f(x)^{p(x)} d x \leq C\left(\int_{\Omega}|f(x)|^{p(x)} d x\right)+\int_{\Omega} S(x) d x \leq C .
$$

This completes the proof.

Our original goal in writing this paper was to provide a proof of Theorem 1.3 which included the case $\alpha=0$; in other words, we wanted to give a unified proof of Theorems 1.1 and 1.3, rather than use the former to prove the latter. In light of (4.2) we made the following conjecture.

Conjecture 4.2. Let $\Omega, \alpha, p(\cdot), q(\cdot)$ be as in Theorem 1.3. If $f$ is such that $|f|_{p(\cdot), \Omega} \leq 1$, then for all $x \in \Omega$,

$$
M_{\alpha} f(x)^{q(x)} \leq C M_{\alpha}\left(|f(\cdot)|^{p(\cdot) / p_{-}}\right)(x)^{q_{-}}+S(x)
$$

where $S(\cdot) \in L^{1}\left(\mathbb{R}^{n}\right)$ and $C=C(n, p(\cdot))$.

We are unable to prove this: the proof of Theorem 4.1 below only holds in the case $\alpha=0$. Nevertheless, we still believe that this conjecture or some minor variant of it is true.

Proof of Theorem 4.1. The first part of this proof has some parts in common with the proof of [3, Lemma 2.3]; however, there are significant differences, so for the convenience of the reader we have included all the details.

First note that without loss of generality we may assume that $f$ is nonnegative. If we define $f_{1}=f \chi_{\{x: f(x) \leq 1\}}$ and $f_{2}=f-f_{1}$, then $f_{i}(x) \leq f(x)$, $i=1,2$, and

$$
M f(x)^{p(x)} \leq 2^{p_{+}}\left(M f_{1}(x)^{p(x)}+M f_{2}(x)^{p(x)}\right) .
$$

Therefore, it will suffice to prove that Theorem 4.1 holds in two special cases: when $f(x) \geq 1$ or $f(x)=0$, and when $f(x) \leq 1$. We will treat each case in turn. Throughout this section, let

$$
R(x)=R_{1}(x)=\frac{1}{(e+|x|)^{n}} .
$$

Also define $\bar{p}(x)=p(x) / p_{-}$. Then $\bar{p}(x) \geq 1$, and (1.3) holds with $p$ replaced by $\bar{p}$. 
Proof of Theorem 4.1 when $f(x) \geq 1$ or $f(x)=0$.

Fix $x \in \Omega$, and fix a ball $B$ of radius $r>0$ containing $x$ such that $|B \cap \Omega|>0$. Let $B_{\Omega}=B \cap \Omega$. We will consider three cases.

Case 1: $\boldsymbol{r}<|\boldsymbol{x}| / \mathbf{4}$. By our assumption on $r$, if $y_{1}, y_{2} \in B$, then $\log (e+$ $\left.\left|y_{1}\right|\right) \approx \log \left(e+\left|y_{2}\right|\right)$. Hence, for all $y \in B_{\Omega}$,

$$
0 \leq \bar{p}(y)-\bar{p}_{-}\left(B_{\Omega}\right) \leq \frac{C}{\log (e+|y|)}
$$

Therefore, by Hölder's inequality and by Lemma 2.7, with $r(\cdot)$ replaced by the constant $\bar{p}_{-}\left(B_{\Omega}\right), s(\cdot)$ by $\bar{p}(\cdot)$, and with $z(y)=y, t=1$, we have that

$$
\begin{gathered}
\left(\frac{1}{|B|} \int_{B_{\Omega}} f(y) d y\right)^{p(x)} \leq\left(\frac{1}{|B|} \int_{B_{\Omega}} f(y)^{\bar{p}_{-}\left(B_{\Omega}\right)} d y\right)^{p(x) / \bar{p}_{-}\left(B_{\Omega}\right)} \\
\quad \leq\left(\frac{C}{|B|} \int_{B_{\Omega}} f(y)^{\bar{p}(y)} d y+\frac{1}{|B|} \int_{B_{\Omega}} R(y)^{\bar{p}_{-}\left(B_{\Omega}\right)} d y\right)^{p(x) / \bar{p}_{-}\left(B_{\Omega}\right)} .
\end{gathered}
$$

Since $r<|x| / 4$, if $y \in B, R(y) \leq C R(x)$. Hence,

$$
\leq\left(\frac{C}{|B|} \int_{B_{\Omega}} f(y)^{\bar{p}(y)} d y+C R(x)^{\bar{p}_{-}\left(B_{\Omega}\right)}\right)^{p(x) / \bar{p}_{-}\left(B_{\Omega}\right)} .
$$

Since $p(x) / \bar{p}_{-}\left(B_{\Omega}\right) \leq p_{+}<\infty$,

$$
\leq 2^{p_{+}} C\left(\frac{1}{|B|} \int_{B_{\Omega}} f(y)^{\bar{p}(y)} d y\right)^{p(x) / \bar{p}_{-}\left(B_{\Omega}\right)}+2^{p_{+}} C R(x)^{p(x)} .
$$

Since $p_{-}>1, R(x)^{p(x)} \in L^{1}\left(\mathbb{R}^{n}\right)$. Hence, to complete Case 1 we will show that the first term is dominated by a constant multiple of

$$
\left(\frac{1}{|B|} \int_{B_{\Omega}} f(y)^{\bar{p}(y)} d y\right)^{p_{-}}
$$

We argue as follows:

$$
\begin{aligned}
& \left(\frac{1}{|B|} \int_{B_{\Omega}} f(y)^{\bar{p}(y)} d y\right)^{p(x) / \bar{p}_{-}\left(B_{\Omega}\right)} \\
& =\left(\frac{1}{|B|} \int_{B_{\Omega}} f(y)^{\bar{p}(y)} d y\right)^{p_{-}}\left(\frac{1}{|B|} \int_{B_{\Omega}} f(y)^{\bar{p}(y)} d y\right)^{\left(p(x) / \bar{p}_{-}\left(B_{\Omega}\right)\right)-p_{-}} \\
& =|B|^{-\left[\left(p(x) / \bar{p}_{-}\left(B_{\Omega}\right)\right)-p_{-}\right] / p_{-}} \\
& \quad \times\left(\int_{B_{\Omega}} f(y)^{p(y)} d y\right)^{\left[\left(p(x) / \bar{p}_{-}\left(B_{\Omega}\right)\right)-p_{-}\right] / p_{-}}\left(\frac{1}{|B|} \int_{B_{\Omega}} f(y)^{\bar{p}(y)} d y\right)^{p_{-}}
\end{aligned}
$$


Observe that

$$
-\frac{1}{p_{-}}\left[\frac{p(x)}{\bar{p}_{-}\left(B_{\Omega}\right)}-p_{-}\right]=p(x)\left[\frac{1}{p(x)}-\frac{1}{p_{-}\left(B_{\Omega}\right)}\right] \leq 0 .
$$

Thus, if $|B| \geq 1$,

$$
|B|^{-\left[\left(p(x) / \bar{p}_{-}\left(B_{\Omega}\right)\right)-p_{-}\right] / p_{-}} \leq 1 .
$$

Otherwise, if $|B| \leq 1$, we have that

$$
p(x)\left[\frac{1}{p(x)}-\frac{1}{p_{-}\left(B_{\Omega}\right)}\right] \geq \frac{p_{+}}{p_{-}^{2}}\left(p_{-}\left(B_{\Omega}\right)-p_{+}\left(B_{\Omega}\right)\right),
$$

so by Lemma 2.5,

$$
|B|^{-\left[\left(p(x) / \bar{p}_{-}\left(B_{\Omega}\right)\right)-p_{-}\right] / p_{-}} \leq|B|^{\left(p_{+} / p_{-}^{2}\right)\left(p_{-}\left(B_{\Omega}\right)-p_{+}\left(B_{\Omega}\right)\right)} \leq C .
$$

Similarly,

$$
\frac{p(x)}{\bar{p}_{-}\left(B_{\Omega}\right)}-p_{-}=\frac{p(x)}{p_{-}\left(B_{\Omega}\right)} p_{-}-p_{-} \geq 0,
$$

so, since $|f|_{p(\cdot), \Omega} \leq 1$,

$$
\left(\int_{B_{\Omega}} f(y)^{p(y)} d y\right)^{\left[\left(p(x) / \bar{p}_{-}\left(B_{\Omega}\right)\right)-p_{-}\right] / p_{-}} \leq 1 .
$$

Combining these three estimates we get inequality (4.1).

Case 2: $|\boldsymbol{x}| \leq \mathbf{1}$ and $\boldsymbol{r} \geq|\boldsymbol{x}| / \mathbf{4}$. As in Case 1, we apply Lemma 2.7. For all $y \in B_{\Omega}$, since $|x| \leq \overline{1}$,

$$
0 \leq \bar{p}(y)-\bar{p}_{-}\left(B_{\Omega}\right) \leq \bar{p}_{+}-\bar{p}_{-} \leq \frac{C}{\log (e+|x|)} .
$$

Therefore, by Hölder's inequality and by Lemma 2.7, with $r(\cdot)$ replaced by the constant $\bar{p}_{-}\left(B_{\Omega}\right), s(\cdot)$ by $\bar{p}(\cdot)$, and with $z(y)=x, t=1$, we have that

$$
\begin{gathered}
\left(\frac{1}{|B|} \int_{B_{\Omega}} f(y) d y\right)^{p(x)} \leq\left(\frac{1}{|B|} \int_{B_{\Omega}} f(y)^{\bar{p}_{-}\left(B_{\Omega}\right)} d y\right)^{p(x) / \bar{p}_{-}\left(B_{\Omega}\right)} \\
\leq\left(\frac{C}{|B|} \int_{B_{\Omega}} f(y)^{\bar{p}(y)} d y+\frac{1}{|B|} \int_{B_{\Omega}} R(x)^{\bar{p}_{-}\left(B_{\Omega}\right)} d y\right)^{p(x) / \bar{p}_{-}\left(B_{\Omega}\right)} .
\end{gathered}
$$

Since $p(x) / \bar{p}_{-}\left(B_{\Omega}\right) \leq p_{+}<\infty$,

$$
\leq 2^{p_{+}}\left(\frac{C}{|B|} \int_{B_{\Omega}} f(y)^{\bar{p}(y)} d y\right)^{p(x) / \bar{p}_{-}\left(B_{\Omega}\right)}+2^{p_{+}} C R(x)^{p(x)} .
$$


We now argue exactly as in Case 1 to show that the integral on the last line is dominated by a constant multiple of

$$
\left(\frac{1}{|B|} \int_{B_{\Omega}} f(y)^{\bar{p}(y)} d y\right)^{p_{-}}
$$

Case 3: $|\boldsymbol{x}| \geq \mathbf{1}$ and $\boldsymbol{r} \geq|\boldsymbol{x}| /$ 4. Since $f(x) \geq 1$ or $f(x)=0, p_{-} \geq 1$ and $|f|_{p(\cdot), \Omega} \leq 1$

$$
\begin{aligned}
\left(\frac{1}{|B|} \int_{B_{\Omega}} f(y) d y\right)^{p(x)} & \leq|B|^{-p(x)}\left(\int_{B_{\Omega}} f(y)^{p(y)} d y\right)^{p(x)} \\
& \leq|B|^{-p(x)} \leq C|x|^{-n p(x)} \leq C R(x)^{p(x)} .
\end{aligned}
$$

This completes the proof of inequality (4.1) when $f(x) \geq 1$ or $f(x)=0$.

\section{Proof of Theorem 4.1 when $f(x) \leq 1$.}

In the previous argument we only used the fact that $f(x) \geq 1$ in Case 3 . Therefore, here it will suffice to fix $x \in \Omega,|x| \geq 1$, and a ball $B$ containing $x$ with radius $r \geq|x| / 4$, and prove that

$$
\left(\frac{1}{|B|} \int_{B_{\Omega}} f(y) d y\right)^{p(x)} \leq C\left(\frac{1}{|B|} \int_{B_{\Omega}} f(y)^{\bar{p}(y)} d y\right)^{p_{-}}+S(x),
$$

where $S(\cdot)$ is some function in $L^{1}$.

Since $p(x) \leq p_{+}<\infty$,

$$
\begin{aligned}
\left(\frac{1}{|B|}\right. & \left.\int_{B_{\Omega}} f(y) d y\right)^{p(x)} \\
& \leq 2^{p_{+}}\left(\frac{1}{|B|} \int_{B_{\Omega} \cap B_{|x|}(0)} f(y) d y\right)^{p(x)}+2^{p_{+}}\left(\frac{1}{|B|} \int_{B_{\Omega} \backslash B_{|x|}(0)} f(y) d y\right)^{p(x)} . \\
& =I_{1}+I_{2} .
\end{aligned}
$$

We will estimate each term separately. To estimate $I_{2}$, we will give an argument very similar to that in Case 2 above. Let $E=B_{\Omega} \backslash B_{|x|}(0)$. Then, if $w, z \in E$, by condition (1.3),

$$
|\bar{p}(w)-\bar{p}(z)| \leq \frac{C}{\log (e+|x|)} ;
$$

as a consequence, for all $y \in E$,

$$
0 \leq \bar{p}(y)-\bar{p}_{-}(E) \leq \bar{p}_{+}(E)-\bar{p}_{-}(E) \leq \frac{C}{\log (e+|x|)}
$$


Therefore, by Hölder's inequality and by Lemma 2.7, with $r(\cdot)$ replaced by $\bar{p}_{-}(E)$, and $s(\cdot)$ by $\bar{p}(\cdot)$, and with $z(y)=x, t=1$, we have that

$$
\begin{gathered}
\left(\frac{1}{|B|} \int_{E} f(y) d y\right)^{p(x)} \leq\left(\frac{1}{|B|} \int_{E} f(y)^{\bar{p}_{-}(E)} d y\right)^{p(x) / \bar{p}_{-}(E)} \\
\quad \leq\left(\frac{C}{|B|} \int_{E} f(y)^{\bar{p}(y)} d y+\frac{1}{|B|} \int_{E} R(x)^{\bar{p}_{-}(E)} d y\right)^{p(x) / \bar{p}_{-}(E)} .
\end{gathered}
$$

Since $p(x) / \bar{p}_{-}(E) \leq p_{+}<\infty$ and $E \subset B_{\Omega}$,

$$
\leq 2^{p_{+}}\left(\frac{C}{|B|} \int_{B_{\Omega}} f(y)^{\bar{p}(y)} d y\right)^{p(x) / \bar{p}_{-}(E)}+2^{p_{+}} R(x)^{p(x)} .
$$

We estimate the first term exactly as we did for the corresponding integral in Case 1 in the above argument, using the fact that $\bar{p}(x) \geq \bar{p}_{-}(E)$. Therefore, we have shown that

$$
I_{2}=\left(\frac{1}{|B|} \int_{B_{\Omega} \backslash B_{|x|}(0)} f(y) d y\right)^{p(x)} \leq C\left(\frac{1}{|B|} \int_{B_{\Omega}} f(y)^{\bar{p}(y)} d y\right)^{p_{-}}+C R(x)^{p(x)}
$$

We now estimate $I_{1}$. Let $F=B_{|x|}(0) \cap B_{\Omega}$. Then for all $y \in F$, by inequality (1.3),

$$
|\bar{p}(x)-\bar{p}(y)| \leq \frac{C}{\log (e+|y|)} .
$$

Therefore, by Hölder's inequality and Lemma 2.8 with $r(\cdot)=\bar{p}(x), s(\cdot)=$ $\bar{p}(\cdot), t=1$,

$$
\begin{aligned}
& \left(\frac{1}{|B|} \int_{F} f(y) d y\right)^{p(x)} \leq\left(\frac{1}{|B|} \int_{F} f(y)^{\bar{p}(x)} d y\right)^{p(x) / \bar{p}(x)} \\
& \quad \leq\left(C \frac{1}{|B|} \int_{F} f(y)^{\bar{p}(y)} d y+\frac{1}{|B|} \int_{B_{|x|}(0)} R(y)^{\bar{p}(x)} d y\right)^{p_{-}} \\
& \quad \leq C\left(\frac{1}{|B|} \int_{F} f(y)^{\bar{p}(y)} d y\right)^{p_{-}}+C\left(\frac{1}{|B|} \int_{B_{|x|}(0)} R(y)^{\bar{p}(x)} d y\right)^{p_{-}}
\end{aligned}
$$

since $r>|x| / 4,\left|B_{|x|}(0)\right| \leq C|B|$; hence,

$$
\leq C\left(\frac{1}{|B|} \int_{B_{\Omega}} f(y)^{\bar{p}(y)} d y\right)^{p_{-}}+C\left(\left|B_{|x|}(0)\right|^{-1} \int_{B_{|x|}(0)} R(y)^{\bar{p}(x)} d y\right)^{p_{-}}
$$


To complete the proof we will show that the second term is dominated by an $L^{1}$ function. Fix $r, 1<r<p_{-}$. Then by Hölder's inequality,

$$
\left(\left|B_{|x|}(0)\right|^{-1} \int_{B_{|x|}(0)} R(y)^{\bar{p}(x)} d y\right)^{p_{-}} \leq\left|B_{|x|}(0)\right|^{-p_{-} / r}\left(\int_{B_{|x|}(0)} R(y)^{\bar{p}(x) r} d y\right)^{p_{-} / r}
$$

Since $\bar{p}(x) r \geq \bar{p}_{-} r>1$ and $R(y) \leq 1$,

$$
\int_{B_{|x|}(0)} R(y)^{\bar{p}(x) r} d y \leq \int_{B_{|x|}(0)} R(y)^{\bar{p}_{-} r} d y \leq C .
$$

Furthermore, since $|x| \geq 1$,

$$
\left|B_{|x|}(0)\right|^{-p_{-} / r} \leq C(e+|x|)^{-n p_{-} / r}=C R_{p_{-} / r}(x),
$$

and the last function is in $L^{1}$ since $p_{-}>r$.

\section{Proof of Theorem 1.6}

In this section we prove Theorem 1.6. We will first prove it in the special case $\alpha=0$, and then prove the general case.

Fix $f \in L^{p(\cdot)}(\Omega)$; without loss of generality we may assume that $\|f\|_{p(\cdot), \Omega}=1$. Then by Lemma $2.2,|f|_{p(\cdot), \Omega} \leq 1$. Fix $t>0$. Then again by Lemma 2.2 it will suffice to prove that

$$
\int_{\Omega}\left(t \chi_{\{M f>t\}}(x)\right)^{p(x)} d x=\int_{\{M f(x)>t\}} t^{p(x)} d x \leq C .
$$

For each $N>0$ define $f_{N}=f \chi_{B_{N}(0)}$. Then for each $N$ the set $\left\{M f_{N}(x)>t\right\}$ is bounded, and by the monotone convergence theorem,

$$
\int_{\{M f(x)>t\}} t^{p(x)} d x=\lim _{N \rightarrow \infty} \int_{\left\{M f_{N}(x)>t\right\}} t^{p(x)} d x .
$$

Therefore, it will suffice to prove that

$$
\int_{\left\{M f_{N}(x)>t\right\}} t^{p(x)} d x \leq C
$$

where $C$ is independent of $N$.

Fix $N>0$. For each $x \in\left\{M f_{N}(x)>t\right\}$ there exists a ball $B_{x}$ containing $x$ such that

$$
\frac{2^{n}}{\left|2 B_{x}\right|} \int_{2 B_{x} \cap \Omega}|f(y)| d y \geq \frac{1}{\left|B_{x}\right|} \int_{B_{x} \cap \Omega}\left|f_{N}(y)\right| d y>t .
$$


Therefore, by the Besicovitch covering lemma (see de Guzmán [15]), there exists a countable subset $\left\{B_{k}\right\}$ of $\left\{B_{x}\right\}$ such that

$$
\left\{M f_{N}(x)>t\right\} \subset \bigcup_{k} 2 B_{k}
$$

and such that the balls $\left\{2 B_{k}\right\}$ have finite overlap uniformly bounded by a constant depending only on the dimension. Hence,

$$
\begin{aligned}
\int_{\left\{M f_{N}(x)>t\right\}} t^{p(x)} d x & \leq \sum_{k} \int_{2 B_{k} \cap \Omega} t^{p(x)} d x \\
& \leq 2^{n p_{+}} \sum_{k} \int_{2 B_{k} \cap \Omega}\left(\frac{1}{\left|2 B_{k}\right|} \int_{2 B_{k} \cap \Omega}|f(y)| d y\right)^{p(x)} d x
\end{aligned}
$$

by Theorem 4.1 , since $p_{-}=1$,

$$
\begin{aligned}
& \leq C \sum_{k} \int_{2 B_{k} \cap \Omega}\left[\frac{1}{\left|2 B_{k}\right|} \int_{2 B_{k} \cap \Omega}|f(y)|^{p(y)} d y+S(x)\right] d x \\
& \leq C \sum_{k} \int_{2 B_{k} \cap \Omega}|f(y)|^{p(y)} d y+\int_{2 B_{k} \cap \Omega} S(x) d x .
\end{aligned}
$$

Since the balls have uniformly bounded finite overlap,

$$
\begin{aligned}
& \leq C \int_{\Omega}|f(y)|^{p(y)} d y+\int_{\Omega} S(x) d x \\
& \leq C .
\end{aligned}
$$

This completes the proof when $\alpha=0$.

Now fix $\alpha, 0<\alpha<n$, and fix $f \in L^{p(\cdot)}(\Omega)$; without loss of generality we may assume that $\|f\|_{p(\cdot), \Omega}=1$. Define $f=f_{1}+f_{2}$, where $f_{1}=f \chi_{\{|f| \geq 1\}}$ and $f_{2}=f \chi_{\{|f|<1\}}$. Then $M_{\alpha} f(x) \leq M_{\alpha} f_{1}(x)+M_{\alpha} f_{2}(x)$, so

$$
\chi_{\left\{M_{\alpha} f(x)>t\right\}} \leq \chi_{\left\{M_{\alpha} f_{1}(x)>t / 2\right\}}+\chi_{\left\{M_{\alpha} f_{2}(x)>t / 2\right\}} .
$$

Therefore, by Propositions 3.1 and 3.2,

$$
\begin{aligned}
t \| \chi_{\left\{M_{\alpha} f>t\right\}} & \left\|_{q(\cdot), \Omega} \leq t\right\| \chi_{\left\{M_{\alpha} f_{1}>t / 2\right\}}\left\|_{q(\cdot), \Omega}+t\right\| \chi_{\left\{M_{\alpha} f_{2}>t / 2\right\}} \|_{q(\cdot), \Omega} \\
& \leq t\left\|\chi_{\left\{M f_{1}(x)^{p(x) / q(x)}>t / 2 C\right\}}\right\|_{q(\cdot), \Omega}+t\left\|\chi_{\left\{M f_{2}(x)^{p(x) / I_{q}(x)}>t / 2 C\right\}}\right\|_{q(\cdot), \Omega} .
\end{aligned}
$$

We can estimate each of the last two terms using an argument essentially the same as in the case when $\alpha=0$. We consider the first. As above, it will suffice to show that

$$
\int_{\left\{M f_{N}(x)^{p(x) / q(x)}>t / 2 C\right\}} t^{q(x)} d x \leq C
$$

where $C$ is independent of $N$. 
But for each $x \in\left\{M f_{N}(x)^{p(x) / q(x)}>t / 2 C\right\}$ there exists a ball $B_{x}$ containing $x$ such that

$$
\left(\frac{2^{n}}{\left|2 B_{x}\right|} \int_{2 B_{x} \cap \Omega}|f(y)| d y\right)^{p(x) / q(x)} \geq\left(\frac{1}{\left|B_{x}\right|} \int_{B_{x} \cap \Omega}\left|f_{N}(y)\right| d y\right)^{p(x) / q(x)}>t / 2 C .
$$

Since $q_{+}<\infty$ the proof now proceeds exactly as it did above.

To estimate the second term we need to show that

$$
\int_{\left\{M f_{N}(x)^{p(x) / I_{q}(x)}>t / 2 C\right\}} t^{q(x)} d x \leq C
$$

where $C$ is independent of $N$. However, arguing exactly as we did at the end of the proof of Theorem 1.3 we have (with the same notation as used there) that

$$
\begin{aligned}
\int_{\left\{M f_{N}(x)^{p(x) / I_{q}(x)}>t / 2 C\right\}} t^{q(x)} d x \leq & C \int_{\left\{M f_{N}(x)^{p(x) / I_{q}(x)}>t / 2 C\right\}} t^{I_{q}(x)} d x \\
& +C \int_{\Omega} R_{2 q_{+} / q_{-}}(x)^{r_{-}} d x
\end{aligned}
$$

The proof now proceeds as it does for the first term.

This completes the proof of Theorem 1.6.

\section{Proof of Theorems 1.8 and $\mathbf{1 . 9}$}

The proofs of Theorems 1.8 and 1.9 depend on an inequality due to Welland [30].

Lemma 6.1. Given $\alpha, 0<\alpha<n$, fix $\epsilon, 0<\epsilon<\max (\alpha, n-\alpha)$. Then there exists a constant $C=C(\alpha, n, \epsilon)$ such that for all $f \in L_{l o c}^{1}\left(\mathbb{R}^{n}\right)$ and all $x \in \mathbb{R}^{n}$,

$$
\left|I_{\alpha} f(x)\right| \leq C\left[M_{\alpha-\epsilon} f(x)\right]^{1 / 2}\left[M_{\alpha+\epsilon} f(x)\right]^{1 / 2} .
$$

Remark 6.2. In [30], Lemma 6.1 is not enunciated as a separate result, but is part of a longer proof. In the proof it is assumed that $\epsilon>0$ is small, but this is a technical hypothesis related to the larger theorem, and is not used to prove inequality (6.1). However, below we will assume that $\epsilon$ is close to 0 .

Proof of Theorem 1.8. Fix $f \in L^{p(\cdot)}(\Omega)$; without loss of generality we may assume that $\|f\|_{p(\cdot), \Omega}=1$. Since $q_{+}<\infty$, by Lemma 2.2 it will suffice to prove that $\left|I_{\alpha} f\right|_{q(\cdot), \Omega} \leq C$. 
Fix $\epsilon, 0<\epsilon<\max (\alpha, n-\alpha)$, such that

$$
\frac{2}{\frac{\epsilon q_{+}}{n}+1}>1
$$

and define $r(\cdot): \Omega \rightarrow[1, \infty)$ by

$$
r(x)=\frac{2}{\frac{\epsilon q(x)}{n}+1} .
$$

Then by (6.2) we have $r_{-}>1$. Moreover, by elementary algebra, for all $x \in \Omega$,

$$
\begin{aligned}
& \frac{1}{p(x)}-\frac{1}{\frac{r(x) q(x)}{2}}=\frac{\alpha-\epsilon}{n} \\
& \frac{1}{p(x)}-\frac{1}{\frac{r^{\prime}(x) q(x)}{2}}=\frac{\alpha+\epsilon}{n} .
\end{aligned}
$$

If we raise both sides of (6.1) to the power $q(x)$ and integrate over $\Omega$ we get

$$
\int_{\Omega}\left|I_{\alpha} f(x)\right|^{q(x)} d x \leq C \int_{\Omega}\left[M_{\alpha-\epsilon} f(x)\right]^{q(x) / 2}\left[M_{\alpha+\epsilon} f(x)\right]^{q(x) / 2} d x .
$$

If we apply Hölder's inequality for variable $L^{p}$ spaces with exponents $r(\cdot)$ and $r^{\prime}(\cdot)$ (Lemma 2.3), then

$$
\int_{\Omega}\left|I_{\alpha} f(x)\right|^{q(x)} d x \leq C\left\|\left[M_{\alpha-\epsilon} f(\cdot)\right]^{q(\cdot) / 2}\right\|_{r(\cdot), \Omega} \times\left\|\left[M_{\alpha+\epsilon} f(\cdot)\right]^{q(\cdot) / 2}\right\|_{r^{\prime}(\cdot), \Omega}
$$

To complete the proof we will estimate each factor on the righthand side. Without loss of generality we may assume that each is greater than 1 , since otherwise there is nothing to prove. In this case, in the definition of each norm we may assume that the infimum is taken over values of $\lambda$ greater than 1. But then, since for all $x \in \Omega$ and $\lambda>1, \lambda^{2 / q(x)} \geq \lambda^{2 / q_{+}}$, we have that

$$
\begin{aligned}
\int_{\Omega}\left(\frac{\left[M_{\alpha-\epsilon} f(x)\right]^{q(x) / 2}}{\lambda}\right)^{r(x)} d x & =\int_{\Omega}\left(\frac{M_{\alpha-\epsilon} f(x)}{\lambda^{2 / q(x)}}\right)^{r(x) q(x) / 2} d x \\
& \leq \int_{\Omega}\left(\frac{M_{\alpha-\epsilon} f(x)}{\lambda^{2 / q_{+}}}\right)^{r(x) q(x) / 2} d x .
\end{aligned}
$$

Therefore, by (6.3) and Theorem 1.3,

$$
\left\|\left[M_{\alpha-\epsilon} f\right]^{q(\cdot) / 2}\right\|_{r(\cdot), \Omega} \leq\left\|M_{\alpha-\epsilon} f\right\|_{r(\cdot) q(\cdot) / 2, \Omega}^{q_{+} / 2} \leq C\|f\|_{p(\cdot), \Omega}^{q_{+} / 2} \leq C .
$$

The norm $\left\|\left[M_{\alpha+\epsilon} f(\cdot)\right]^{q(\cdot) / 2}\right\|_{r(\cdot)^{\prime}, \Omega}$ can be estimated in the same way, replacing in the argument above $\alpha-\epsilon$ by $\alpha+\epsilon, r(\cdot)$ by $r^{\prime}(\cdot)$, and using (6.4) instead of (6.3). This completes the proof. 
Proof of Theorem 1.9. Fix $f \in L^{p(\cdot)}(\Omega)$; without loss of generality we may assume that $\|f\|_{p(\cdot), \Omega}=1$. Since $q_{+}<\infty$, by Lemma 2.2 it will suffice to prove that

$$
\int_{\left\{\left|I_{\alpha} f\right|>t\right\}} t^{q(x)} d x \leq C .
$$

Fix $\epsilon, r(\cdot)$ and $r^{\prime}(\cdot)$ as in the proof of Theorem 1.8. Since $r_{-}>1,\left(r^{\prime}\right)_{+}<\infty$. By Lemma 6.1 and Young's inequality, for each $x$,

$$
\begin{aligned}
\left|I_{\alpha} f(x)\right| & \leq C M_{\alpha-\epsilon} f(x)^{1 / 2} M_{\alpha+\epsilon} f(x)^{1 / 2} \\
& \leq \frac{\left[C M_{\alpha-\epsilon} f(x)\right]^{r(x) / 2}}{r(x)}+\frac{\left[C M_{\alpha+\epsilon} f(x)\right]^{r^{\prime}(x) / 2}}{r^{\prime}(x)} \\
& \leq C^{r_{+}} M_{\alpha-\epsilon} f(x)^{r(x) / 2}+C^{\left(r^{\prime}\right)+} M_{\alpha+\epsilon} f(x)^{r^{\prime}(x) / 2} .
\end{aligned}
$$

Therefore,

$$
\int_{\left\{\left|I_{\alpha} f\right|>t\right\}} t^{q(x)} d x \leq \int_{\left\{M_{\alpha-\epsilon} f(x)^{r(x) / 2}>t / 2 C\right\}} t^{q(x)} d x+\int_{\left\{M_{\alpha+\epsilon} f(x)^{r^{\prime}(x) / 2}>t / 2 C\right\}} t^{q(x)} d x .
$$

We estimate each of these integrals as we did in the second half of the proof of Theorem 1.6, using first the exponent pair $p(\cdot), r(\cdot) q(\cdot) / 2$ or the exponent pair $p(\cdot), r^{\prime}(\cdot) q(\cdot) / 2$ to apply Theorem 4.1 and then arguing as before.

\section{A. The Nekvinda Condition}

In the case $\Omega=\mathbb{R}^{n}$, Nekvinda [24] independently proved a more general version of Theorem 1.1. To compare his result to ours, we first must slightly restate one of our hypotheses. If the exponent function $p(\cdot)$ satisfies (1.3), then this is equivalent to the existence of a constant $p_{\infty}$ and a function $\phi(\cdot)$ converging to zero as $|x| \rightarrow \infty$ such that $p(x)=p_{\infty}+\phi(x)$ and

$$
|\phi(x)-\phi(y)| \leq \frac{C}{\log (e+|x|)}, \quad|y| \geq|x| .
$$

Nekvinda replaced this condition with the following: there exists $\beta>0$ such that

$$
\int_{\{x:|\phi(x)|>0\}}|\phi(x)| \beta^{1 /|\phi(x)|} d x<\infty .
$$

Clearly, condition (A.1) is easier to verify than (A.2); on the other hand, (A.2) is better than (A.1): from (A.1) we have that $|\phi(x)| \leq C[\log (e+|x|)]^{-1}$, so if we let $\beta=e^{-k}, k>0$, the integral in (A.2) converges if we choose $k$ sufficiently large. (We remark in passing that this extends [24, Example 2.15] to the case $\beta=1$.) 
But, as the next example shows, the converse is false even for functions which satisfy (1.2).

Example A.1. There exists a function $\phi(\cdot): \mathbb{R} \rightarrow[0,1]$ which satisfies (1.2) and (A.2) but not (A.1).

Proof. We define $\phi(\cdot)$ as follows:

$$
\phi(x)= \begin{cases}\frac{1}{n}-\left|e^{n^{2}}-x\right| & 0 \leq\left|e^{n^{2}}-x\right| \leq \frac{1}{n}, \quad 1 \leq n<\infty \\ 0 & \text { otherwise }\end{cases}
$$

Then we have that

$$
\phi\left(e^{n^{2}}\right)=\frac{1}{n} \approx \frac{n}{\log \left(e+e^{n^{2}}\right)}, \quad \phi\left(e^{n^{2}}+1 / n\right)=0,
$$

so (A.1) cannot hold. On the other hand, it follows immediately from the definition that $\phi$ is Lipschitz, so (1.2) holds. Furthermore, if we let $\beta=e^{-1}$, then

$$
\int_{\left\{\mid e^{\left.n^{2}-x \mid<1 / n\right\}}\right.} \phi(x) e^{-1 / \phi(x)} d x=2 \int_{0}^{1 / n} y e^{-1 / y} d y=2 \int_{n}^{\infty} z^{-3} e^{-z} d z \leq \frac{1}{n^{2} e^{n}} .
$$

Hence,

$$
\int_{\{x: \phi(x)>0\}} \phi(x) e^{-1 / \phi(x)} d x \leq \sum_{n=1}^{\infty} \frac{1}{n^{2} e^{n}}<\infty
$$

Given fairly weak additional hypotheses, (A.2) does imply (A.1): for example, if the least radial majorant of the integrand in (A.2) is dominated by a power function. This is the substance of the next result.

Proposition A.2. Given a bounded function $\phi(\cdot)$, suppose that for some $\beta>0$ there exists $a>0$ such that for all $x \in\{x:|\phi(x)|>0\}$,

$$
|\phi(x)| \beta^{1 /|\phi(x)|} \leq \frac{C}{(e+|x|)^{a}} .
$$

Then (A.1) holds.

Proof. If we rearrange (A.3) and take the logarithm of both sides, we get

$$
a \log (e+|x|) \leq \frac{1}{|\phi(x)|}(C|\phi(x)|-|\phi(x)| \log |\phi(x)|-\log \beta) .
$$

Since $\phi(\cdot)$ is bounded, and since $|t| \log |t|$ is bounded for $t$ close to 0 , it follows that

$$
\log (e+|x|) \leq \frac{C}{|\phi(x)|}
$$

and (A.1) follows immediately. 
Finally, we note that our proof of Theorem 1.1 can be generalized to include additional exponent functions $p(\cdot)$. In the proof given above (or in the proof in [3]), we can replace the function $R(x)=(e+|x|)^{-n}$ with any function $R$ such that $R^{p_{-}} \in L^{1}$, and such that $R$ is roughly constant on balls whose radii are small compared to their distances from the origin-for instance, functions which satisfy

$$
R(y) \approx R(x), \quad \frac{|x|}{2} \leq|y| \leq 2|x| .
$$

Given such an $R$, we then modify condition (1.3) (or (A.1)), replacing $\log (e+|x|)^{-1}$ with $\log (R(x))^{-1}$. If we do this, then the proof goes through with only slight changes. Details are left to the interested reader.

Acknowledgment. The authors would like to thank the anonymous referee, whose valuable comments considerably simplified the proof of Theorem 1.3.

\section{References}

[1] Acerbi, E. And Mingione, G.: Regularity results for stationary electrorheological fluids. Arch. Ration. Mech. Anal. 164 (2002), 213-259.

[2] Cruz-Uribe, D. SFO And Fiorenza, A.: Approximate identities in variable $L^{p}$ spaces. Math. Nachr. 280 (2007), no. 3, 256-270.

[3] Cruz-Uribe, D. SFO, Fiorenza, A. and Neugebauer, C. J.: The maximal function on variable $L^{p}$ spaces. Ann. Acad. Sci. Fenn. Math. 28 (2003), 223-238.

[4] Cruz-Uribe, D. SFO, Fiorenza, A. and Neugebauer, C. J.: Corrections to: "The maximal function on variable $L^{p}$ spaces". Ann. Acad. Sci. Fenn. Math. 29 (2004), 247-249.

[5] Diening, L.: Maximal function on generalized $L^{p(\cdot)}$ spaces. Math. Inequal. Appl. 7 (2004), 245-253.

[6] Diening, L.: Riesz potential and Sobolev embeddings on generalized Lebesgue and Sobolev spaces $L^{p(\cdot)}$ and $W^{k, p(\cdot)}$. Math. Nachr. 268 (2004), $31-43$.

[7] Diening, L.: Maximal function on Musielak-Orlicz spaces and generalized Lebesgue spaces. Bull. Sci. Math. 129 (2005), no. 8, 657-700.

[8] Diening, L., Hëstö, P. And Nekvinda, A.: Open problems in variable exponent Lebesgue and Sobolev spaces. In Function Spaces, Differential Operators and Nonlinear Analysis (Milovy, Czech Republic, 2004), 38-58. Academy of Sciences of the Czech Republic, Prague, 2005.

[9] Edmunds, D. And RÁkosník, J.: Sobolev embeddings with variable exponent. Studia Math. 143 (2000), 267-293.

[10] Edmunds, D. And RÁkosník, J.: Sobolev embeddings with variable exponent II. Math. Nachr. 246/247 (2002), 53-67. 
[11] FAn, X. AND ZhaO, D.: On the spaces $L^{p(x)}(\Omega)$ and $W^{m, p(x)}(\Omega)$. J. Math. Anal. Appl. 263 (2001), 424-446.

[12] Fusco, N. And Sbordone, C.: Some remarks on the regularity of minima of anisotropic integrals. Comm. Partial Differential Equations 18 (1993), 153-167.

[13] Giaquinta, M.: Growth conditions and regularity, a counterexample. Manuscripta Math. 59 (1987), 245-248.

[14] Gilbarg, D. and Trudinger, N. S.: Elliptic partial differential equations of second order. Second edition. Grundlehren der Mathematischen Wissenschaften 224. Springer-Verlag, Berlin, 1983.

[15] Guzmán, M. DE: Differentiation of integrals in $\mathbb{R}^{n}$. Lecture Notes in Mathematics 481. Springer-Verlag, New York, 1975.

[16] Hedberg, L.: On certain convolution inequalities. Proc. Amer. Math. Soc. 36 (1972), 505-510.

[17] Hudzik, H.: On generalized Orlicz-Sobolev space. Funct. Approximatio Comment. Math. 4 (1976), 37-51.

[18] Kokilashvili, V. And Samko, S.: Maximal and fractional operators in weighted $L^{p(x)}$ spaces. Rev. Mat. Iberoamericana 20 (2004), no. 2, 493-515.

[19] Kokilashvili, V. and Samko, S.: On Sobolev theorem for Riesz-type potentials in Lebesgue spaces with variable exponent. Z. Anal. Anwendungen 22 (2003), no. 4, 899-910.

[20] Kováčik, O. And RÁkosník, J.: On spaces $L^{p(x)}$ and $W^{k, p(x)}$. Czechoslovak Math. J. 41(116) (1991), no. 4, 592-618.

[21] Lerner, A.: Some remarks on the Hardy-Littlewood maximal function on variable $L^{p}$ spaces. Math. Z. 251 (2005), no. 3, 509-521.

[22] Marcellini, P.: Regularity and existence of solutions of elliptic equations with $p, q$-growth conditions. J. Differential Equations 90 (1991), 1-30.

[23] Musielak, J.: Orlicz spaces and modular spaces. Lecture Notes in Mathematics 1034. Springer-Verlag, Berlin, 1983.

[24] Nekvinda, A.: Hardy-Littlewood maximal operator on $L^{p(x)}\left(\mathbb{R}^{n}\right)$. Math. Inequal. Appl. 7 (2004), 255-265.

[25] PiCK, L. AND RŮŽIČKA, M.: An example of a space $L^{p(x)}$ on which the Hardy-Littlewood maximal operator is not bounded. Expo. Math. 19 (2001), no. 4, 369-371.

[26] RŮŽIČKA, M.: Electrorheological fluids: modeling and mathematical theory. Lecture Notes in Mathematics 1748. Springer-Verlag, Berlin, 2000.

[27] Samko, S. G.: Convolution and potential type operators in $L^{p(x)}\left(\mathbb{R}^{n}\right)$. Integral Transform. Spec. Funct. 7 (1998), 261-284.

[28] Samko, S. G.: Density $C_{0}^{\infty}\left(\mathbb{R}^{n}\right)$ in the generalized Sobolev spaces $W^{m, p(x)}\left(\mathbb{R}^{n}\right)$. (Russian) Dokl. Akad. Nauk 369 (1999), 451-454. Translated in Russian Acad. Sci. Dokl. Math. 60 (1999), 382-385. 
[29] Stein, E.M.: Singular integrals and differentiability properties of functions. Princeton Mathematical Series 30. Princeton University Press, Princeton, N. J., 1970.

[30] Welland, G.V.: Weighted norm inequalities for fractional integrals. Proc. Amer. Math. Soc. 51 (1975), 143-148.

[31] Zhikov, V.V.: Averaging of functionals of the calculus of variations and elasticity theory. (Russian) Izv. Akad. Nauk SSSR Ser. Mat. 50 (1986), $675-710,877$.

[32] Ziemer, W.P.: Weakly differentiable functions. Sobolev spaces and functions of bounded variation. Graduate Texts in Mathematics 120. SpringerVerlag, New-York, 1989.

Recibido: 16 de junio de 2005

Revisado: 29 de marzo de 2006

Claudia Capone

Istituto per le Applicazioni del Calcolo "Mauro Picone", sezione di Napoli Consiglio Nazionale delle Ricerche via Pietro Castellino, 111 I-80131 Napoli, Italy capone@na.iac.cnr.it

David Cruz-Uribe, SFO

Dept. of Mathematics Trinity College

Hartford, CT 06106-3100, USA david.cruzuribe@trincoll.edu

Alberto Fiorenza

Dipartimento di Costruzioni e Metodi Matematici in Architettura Universitá di Napoli Via Monteoliveto, 3 I-80134 Napoli, Italy

and

Istituto per le Applicazioni del Calcolo "Mauro Picone", sezione di Napoli Consiglio Nazionale delle Ricerche via Pietro Castellino, 111 I-80131 Napoli, Italy fiorenza@unina.it 